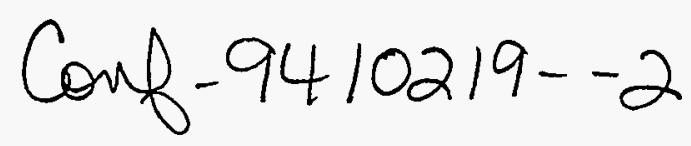

LA-UR- $94 \div 396$

Title:

\title{
SMULATION AND STEERING IN THE INTERTANK MATCHING SECTION OF THE GROUND TEST ACCELERATOR
}

Author(s):

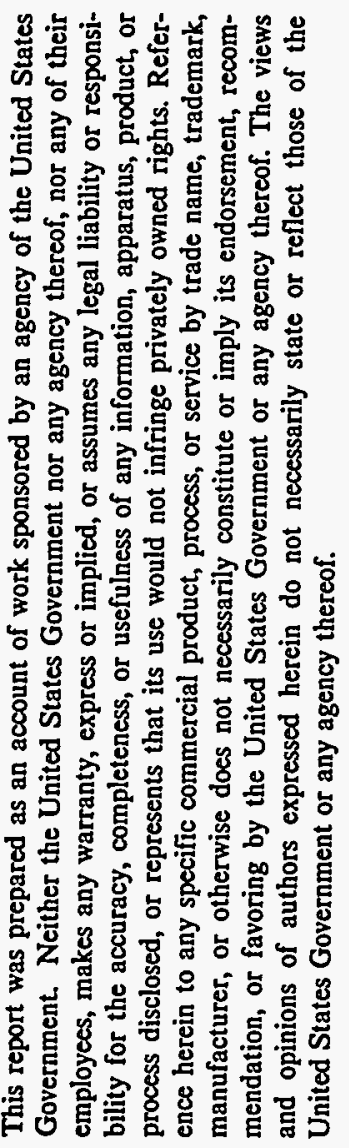

Submitted to:

Beam Instrumentation Workshop - 94 (BIW-94)

Vancouver, B.C., Canada

October 2-6, 1994

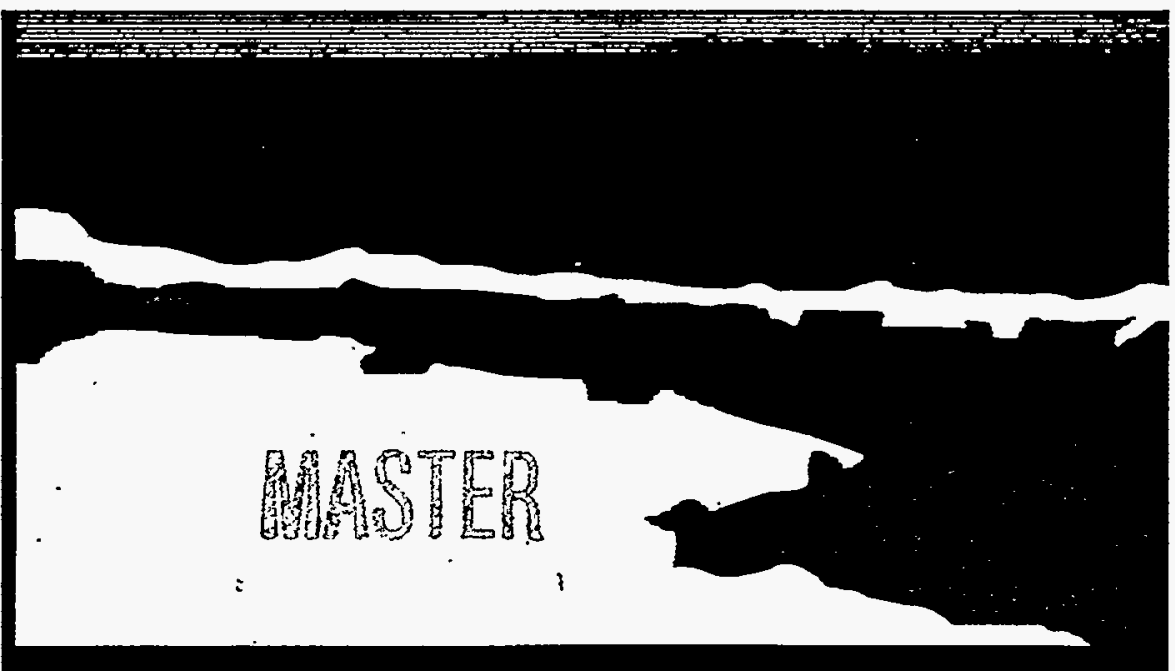

Los Alamos National Laboratory, an affirmative action/equal opportunity employer, is operated by the University of Califomia for the U.S. Department of Energy under contract W-7405-ENG-36. By acceptance of this article, the publisher recognizes that the U.S. Government retains a nonexclusive, royalty-free license to publish or reproduce the published form of this contribution, or to allow others to do so, for U.S. Govemment purposes. The Los Alamos National Laboratory requests that the publisher identify this article as work performed under the auspices of the U.S. Department of Energy.

$$
\text { Se }
$$




\section{DISCLAIMER}

Portions of this document may be illegible in electronic image products. Images are produced from the best available original document. 


\title{
Simulation and steering in the Intertank Matching Section of the Ground Test Accelerator*
}

\author{
V. W. Yuan, G. O. Bolme, J. L. Erickson ${ }^{\dagger}$, K. F. Johnson, \\ C. T. Mottershead, O. R. Sander, M. T. Smith \\ Los Alamos National Laboratory, Los Alamos NM 87545
}

\section{Introduction}

The Intertank Matching Section (IMS) of the Ground Test Accelerator (GTA) is a short $(36 \mathrm{~cm})$ beamline designed to match the Radio Frequency Quadrupole (RFQ) exit beam into the first Drift Tube LINAC (DTL) tank. The IMS contains two steering quadrupoles (SMQs) and four variable-field focussing quads (VFQs). The SMQs are fixed strength permanent magnet quadrupoles on mechanical actuators capable of transverse movement for the purpose of steering the beam. The upsteam and downstream steering quadrupoles are labelled SMQ1 and SMQ4 respectively. Also contained in the IMS are two $\mathrm{RF}$ cavities for longitudinal matching. A schematic of the IMS beam line is shown in figure 1.

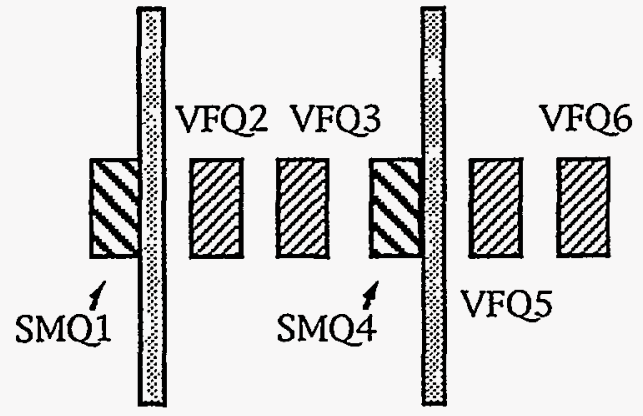

RF1

RF2
Figure.1. Schematic diagram of IMS

The settings of the Variable-field quadrupoles, named VFQ2, VFQ3, VFQ5, and VFQ6, are called the "tune" of the IMS, expressed as the GL product (in Tesla) of each VFQ. The tunes of the IMS were chosen for DTL matching, but have a significant impact on the steering sensitivities. A diagnostics plate containing a slit and collector diagnostic (ES5) was located just downstream of the IMS and was used for measuring changes in positional and angular distributions of the beam when SMQ positions were varied. These measured values are compared with the changes predicted by TRACE3D(1) simulations.

*Work supported and funded by the US Department of Defense, Army Strategic Defense Command, under the auspices of the Department of Energy.

Industrial partner, Grumman Energy Systems Division, Bethpage, NY 


\section{Steering Coefficients for a Single Quad}

The transport through a quadrupole with transfer matrix $Q$, displaced by a steering offset $+s$ may be written as

$$
\left[\begin{array}{c}
x_{e}-s \\
p_{e}
\end{array}\right]=\left[\begin{array}{ll}
Q_{11} & Q_{12} \\
Q_{21} & Q_{22}
\end{array}\right]\left[\begin{array}{c}
x_{0}-s \\
p_{0}
\end{array}\right] \text { or } \begin{aligned}
& x_{e}=Q_{11} x_{0}+Q_{12} p_{0}+\left(1-Q_{11}\right) s \\
& p_{e}=Q_{21} x_{0}+Q_{22} p_{0}-Q_{21} s
\end{aligned}
$$

where $\left(x_{0}, p_{0}\right)$ are the coordinates of the centroid ray entering the quad and $\left(x_{e}, p_{e}\right)$ are its coordinates exiting the quad. The terms that remain when $s=0$ are the normal application of the transfer matrix of the quadrupole.

We are normally interested in the beam centroid steering movements at a particular downstream monitor location. The beam coordinates at the monitor $\left(x_{m}, p_{m}\right)$ are obtained by propagating the steering-quad exit coordinates $\left(x_{e}, p_{e}\right)$ through the R-matrix between the two points:

$$
\left[\begin{array}{l}
x_{m} \\
p_{m}
\end{array}\right]=\left[\begin{array}{ll}
R_{11} & R_{12} \\
R_{21} & R_{22}
\end{array}\right]\left[\begin{array}{l}
x_{e} \\
p_{e}
\end{array}\right]
$$

or

$$
\begin{aligned}
& x_{m}=(R Q)_{11} x_{0}+(R Q)_{12} p_{0}+\left\{R_{11}\left(1-Q_{11}\right)-R_{12} Q_{21}\right\} s \\
& p_{m}=(R Q)_{21} x_{0}+(R Q)_{22} p_{0}+\left\{R_{21}\left(1-Q_{11}\right)-R_{22} Q_{21}\right\} s
\end{aligned}
$$

From this we read off the steering coefficients for the monitor in question:

$$
\begin{aligned}
& C \equiv \frac{\partial x_{m}}{\partial s}=R_{11}\left(1-Q_{11}\right)-R_{12} Q_{21} \\
& A \equiv \frac{\partial p_{m}}{\partial s}=R_{21}\left(1-Q_{11}\right)-R_{22} Q_{21} .
\end{aligned}
$$

Again, for $s=0$, we are left with just the usual first order propagation of the incoming beam, beginning at the input surface of the steering quad.

\section{Case of Two Steerers}

Now what happens when a second steering quad (SMQ4) is added downstream of the first one (SMQ1)? The result becomes more complicated, but still remains linear in the steering offsets. To see this, consider Eq.1 applied to the second steerer, but with the input coordinates replaced by the $\left(x_{m}, p_{m}\right)$ resulting from the first steerer. The result is of the same matrix form as Eq. 1 , but with additional terms linear in the offset $s_{4}$ of the second quad. The R-matrix can be redefined to carry the beam from wherever $\left(x_{0}, p_{0}\right)$ is specified to the point of interest $\left(x_{f}, p_{f}\right)$. The general expression for the final beam centroid coordinates may then be written

$$
\left[\begin{array}{l}
x_{f} \\
p_{f}
\end{array}\right]=\left[\begin{array}{ll}
R_{11} & R_{12} \\
R_{21} & R_{22}
\end{array}\right]\left[\begin{array}{l}
x_{0} \\
p_{0}
\end{array}\right]+\left[\begin{array}{ll}
C_{1} & C_{4} \\
A_{1} & A_{4}
\end{array}\right]\left[\begin{array}{l}
s_{1} \\
s_{4}
\end{array}\right]
$$


where the displacement of the first quad is now labelled $s_{1}$. The final beam coordinates are thus seen to be a sum of two contributions. The first depends only on the incoming beam $\left(x_{0}, p_{0}\right)$ as propagated by the usual R-matrix. The second depends only on the steering offsets $\left(s_{1}, s_{4}\right)$ as propagated by a new steering, or S-matrix, built as shown from the steering sensitivity coefficients

$$
C_{k} \equiv \frac{\partial x_{f}}{\partial s_{k}} \text { and } A_{k} \equiv \frac{\partial p_{f}}{\partial s_{k}}
$$

There are, of course, corresponding expressions in the y-plane.

\section{Effective pivot points}

The optics beyond VFQ6 is simply a drift space in which particle trajectories are straight lines at a constant angle. The angular sensitivity coefficients $A_{k}$ are therefore independent of longitudinal coordinate $z$ in the drift space, and thus the same for all monitoring points beyond VFQ6. The position steering coefficients $C_{k}$ have a linear $z$ dependence in a drift, which may be seen by transporting the output coordinates from Eq. 4 through an additional drift: from the reference point $z_{f}$ where $C_{k}$ and $A_{k}$ were evaluated to the final point of interest at $z$

$$
\left[\begin{array}{l}
x(z) \\
p(z)
\end{array}\right]=\left[\begin{array}{cc}
1 & z-z_{f} \\
0 & 1
\end{array}\right]\left\{\left[\begin{array}{ll}
R_{11} & R_{12} \\
R_{21} & R_{22}
\end{array}\right]\left[\begin{array}{l}
x_{0} \\
p_{0}
\end{array}\right]+\left[\begin{array}{ll}
C_{1} & C_{4} \\
A_{1} & A_{4}
\end{array}\right]\left[\begin{array}{l}
s_{1} \\
s_{4}
\end{array}\right]\right\}
$$

When this is multiplied out one sees that $C_{k}(z)=C_{k}+\left(z-z_{f}\right) A_{k}$ is linear in $z$, while $A_{k}(z)=A_{k}$ is constant. The position coefficient $C_{k}(z)$ passes through zero at $z_{k} \equiv z_{f}-C_{k} / A_{k}$, which we call the effective pivot point for converting centroid angle swings, due to steering-quad movements, into position changes. This means that the steering matrix anywhere in the drift is completely specified by an angular sensitivity $A_{k}$ and a pivot point coordinate $z_{k}$ for each steering quad:

$$
\mathrm{S}(z)=\left[\begin{array}{cc}
\left(z-z_{1}\right) A_{1} & \left(z-z_{4}\right) A_{4} \\
A_{1} & A_{4}
\end{array}\right]
$$

Obviously, a steering quad will not affect the transverse position of the beam at its own pivot point coordinate.

\section{$5 \quad$ Measured steering coefficients}

Steering studies were performed for 3 different tunes of the IMS variablefield quadrupoles. These tunes are designated tune 1 , tune 2 , and tune 3 . Table 1 summarizes the nominal quadrupole field settings for the three different tunes used in the steering studies. 


\begin{tabular}{|c|c|r|r|r|}
\hline Tune & VFQ2 & VFQ3 & VFQ5 & VFQ6 \\
\hline 1 & -5.583 & 4.884 & 6.288 & -6.136 \\
\hline 2 & -4.954 & 4.674 & 5.644 & -5.891 \\
\hline 3 & -4.380 & 5.635 & 5.667 & -6.174 \\
\hline
\end{tabular}

Table 1. Quadrupole (VFQ) field settings of the three IMS tunes used for our experimental measurements. Fields are given in Tesla.

The measured steering coefficients were determined from sets of runs incorporating all three beam tunes and in which the steering quadrupoles were singly displaced along either the $\mathrm{x}$ or $\mathrm{y}$ axis. The resultant changes in beam position and angle were monitored at ES5. For the displacements of each quadrupole, the data were linearly fitted using MINUIT(2), a minimum chi-squared routine, to determine the steering coefficients With separate coefficients for each of the $x$ and $y$ directions, and for position and angle sensitivities, this results in 8 steering coefficients for each tune and 24 coefficients overall for the entire set of 3 beam tunes.

The measured steering coefficients appear along with MINUIT-determined uncertainties in Table 2. These uncertainties were determined by adjusting the error on each individual data point until the fit using MINUIT resulted in a chi-squared of one per degree of freedom (DOF). There is a wide spread in the uncertainty values due in part to the limited number of points that comprised each data set and in part to the mechanical limitation on the range of travel of the ES5 slit and collector. When the steering quadrupoles were displaced and the beam was not centered at ES5, the slit and collector could not always capture the full phase space of the beam, and centroid determination in those cases had to be made based on high level contours of the beam distribution.

\section{Comparison of measured steering coefficients with simulation}

For comparison with measured steering coefficients, a set of coefficients was calculated using the TRACE3D simulation. These calculated coefficients were determined assuming nominal quadrupole field strengths, and they are compared to measured values in Table 2 . In calculating the simulation values, we treated the steering quadrupoles as permanent-magnet quadrupoles (PMQs) with fringe fields extending into the neigboring drift spaces. The VFQs were treated as quadrupole magnets with hard-edged fields that cut off abruptly at the magnets' physical boundaries.(3) The average difference between calculated and measured values is $18 \%$, and accepting the uncertainties of Table 2 , the chi-squared for the comparison of calculated values to measured values is 20/DOF. Such a large chi-squared value is indicative of poor agreement 
Tune 1

Tune 2

\begin{tabular}{|l|r|r|r||r|r|r|}
\hline Coeff & measured & calc & fit & measured & calc & fit \\
\hline CX1 & $-20.50 \pm 0.65$ & -23.08 & -19.90 & $-11.31 \pm 0.51$ & -14.88 & -10.87 \\
\hline CY1 & $4.59 \pm 0.22$ & 3.95 & 4.51 & $4.68 \pm 0.15$ & 3.81 & 4.62 \\
\hline CX4 & $-8.98 \pm 0.58$ & -7.71 & -7.94 & $-10.98 \pm 0.39$ & -10.22 & -10.18 \\
\hline CY4 & $-17.05 \pm 0.77$ & -19.84 & -17.70 & $-15.44 \pm 0.39$ & -16.98 & -15.12 \\
\hline AX1 & $-52.90 \pm 1.81$ & -61.11 & -54.09 & $-30.50 \pm 1.20$ & -40.75 & -31.63 \\
\hline AY1 & $11.51 \pm 0.87$ & 9.70 & 11.73 & $11.24 \pm 0.41$ & 8.54 & 11.43 \\
\hline AX4 & $-19.78 \pm 1.57$ & -17.55 & -18.22 & $-23.86 \pm 0.98$ & -23.71 & -23.71 \\
\hline AY4 & $-51.73 \pm 2.77$ & -60.01 & -53.84 & $-48.37 \pm 1.31$ & -52.05 & -46.64 \\
\hline
\end{tabular}

Tune 3

\begin{tabular}{|r|r|r|r|}
\hline Coeff & measured & calc & fit \\
\hline CX1 & $-11.00 \pm 2.84$ & -15.74 & -11.22 \\
\hline CY1 & $10.74 \pm 0.75$ & 10.05 & 10.80 \\
\hline CX4 & $-10.24 \pm 0.59$ & -11.94 & -11.81 \\
\hline CY4 & $-16.46 \pm 0.76$ & -19.60 & -17.51 \\
\hline AX1 & $-28.05 \pm 6.84$ & -40.54 & -30.32 \\
\hline AY1 & $29.18 \pm 2.45$ & 26.73 & 29.50 \\
\hline AX4 & $-23.00 \pm 1.59$ & -28.01 & -27.78 \\
\hline AY4 & $-50.65 \pm 2.76$ & -58.77 & -52.77 \\
\hline
\end{tabular}

Table 2. Measured, calculated and fit coefficients for Tunes 1,2, and 3. Calculated coefficients are based on the nominal quadrupole fields given in Table 1.

\begin{tabular}{|c|r|r|r|}
\hline magnet & GL (old) & \multicolumn{1}{|c|}{ GL } & \% change \\
\hline SMQ1 & 4.742 & -0.184 & -3.88 \\
\hline VFQ2 & -5.583 & 0.076 & -1.37 \\
\hline VFQ3 & 4.884 & -0.594 & -12.16 \\
\hline SMQ4 & -5.499 & 0.389 & -7.08 \\
\hline VFQ5 & 6.288 & -0.202 & -3.22 \\
\hline VFQ6 & -6.136 & 0.114 & -1.86 \\
\hline
\end{tabular}

Table 3. Changes to quadrupole field values (in Tesla) determined by FELDFIT program when quadrupole strengths are varied to achieve the best match of calculated to measured steering coefficients. 
between measured and calculated values for the uncertainties assumed. Three possible reasons for the disagreement could be: 1) the uncertainties are larger than assumed, 2) the quadrupole field values are not what is assumed, or 3) the simulation model is incorrect.

\section{Fitting program results}

A fitting program, FIELDFIT, was written that allows quadrupole field strengths to differ from their nominal values in order to optimize the agreement of measured to calculated steering coefficients. It therefore searches for a set of deviations in quadrupole field strengths which results in a significant reduction in chi-squared between measured and calculated steering coefficients.

The fitting program FIELDFIT incorporates TRACE3D beam-tracking and matrix propagation routines to calculate steering coefficients. This allows the correct calculation of steering coefficients when more than one of the quad fields are varied. If FIELDFIT is used to fit simultaneously the sets of data for the 3 different tunes, a fit with a much-improved chi-squared of 1.9/DOF can be obtained with the restriction that the same fractional quadrupole deviations are assumed for each tune. The fit values for such a fit are listed in Table 2 under the column heading "fit". The fit-determined steering coefficients have an average of $4.9 \%$ deviation from the measured values. This is approximately 3.5 times smaller than the average $17.7 \%$ deviation of the nominal-field-determined coefficients from the measured values.

The field deviations determined by the fit are listed in Table 3 . The largest two deviations are at the $12.1 \%$ and $7.1 \%$ levels, and the other deviations are at the $3.9 \%, 3.2 \%, 1.9 \%$, and $1.4 \%$ levels. The average deviation is $4.9 \%$ which is 2.5 times larger than the $2 \%$ uncertainty in GL estimated by our magnet mapping group. The largest deviation, $12.1 \%$, corresponds to a field deviation of $.6 \mathrm{~T}$ which seems unreasonably large. It is interesting that all the field deviations determined by the fit are in the direction to decrease the magnitude of each field strength.

With a set of programs written using the ADLIB(4) modular format, we estimated the error in each steering coefficient which results from a $2 \%$ standard deviation in each quadrupole field value. For each of the three IMS tunes, a Gaussian random number generator was used to produce a file of 2000 possible samples of the tune, such that the ensemble average strength of each quad was its nominal value, with a standard deviation of $2 \%$ of the nominal value. The result of these Gaussian field-strength errors is an approximate $9 \%$ average uncertainty in the steering coefficients. We see from the FIELDFIT analysis that field deviations which average $4.9 \%$ and which contain individual deviations of as large as $12 \%$ will change the steering coefficients by an average of approximately $13 \%$. 


\section{Conclusions}

A comparison of measured to calculated steering coefficients has been made for data taken in 3 different tunes of the IMS transport line. Using a TRACE3Dbased calculation, calculated values differ from measured values by an average 17.7\%. Estimated uncertainties in the measured values are too small to explain the observed discrepancy. Changes to the effective field strengths of the quadrupoles were found that result in a significant improvement between measured and calculated values. The field strength deviations required, however, are on average 2.5 times larger than what is estimated by our magnet mapping group as the actual uncertainty of each magnet. The model calculation is not perfect. For instance, we know that the VFQ fringe fields are not correctly modelled by TRACE3D. However, tests with approximations to the field shape effect the results by only $20 \%$. The fact that changing the field strengths of the quadrupoles can significantly improve the agreement between measured and calculated coefficients may well indicate the presence of some other as yet unknown systematic problem with the model calculation.

\section{REFERENCES}

[1] K. R. Crandall and D. P. Rusthoi, "TRACE 3-D Documentation", Los Alamos report LA-UR-90-4146, second edition (1990).

[2] General minimization program contained in CERNLIB codes, CERN, Geneva, Switzerland.

[3] In actuality the VFQ fields have fringe fields that lies somewhere between the field of an electromagnet and that of a permanent-magnet quadrupole (PMQ). This type of hybrid fringe field is not expressly treated in TRACE3D. However an approximation to this fringe field shape can be made by treating the VFQ as a PMQ with an artificially small inner-bore radius. Using such an approximation results in a $20 \% \mathrm{im}-$ provement in the agreement between the measured and calculated steering coefficients for the nominal quadrupole fields.

[4] C. T. Mottershead, "ADLIB- A Simple Database Framework for Beamline Codes", Computational Accelerator Physics, AIP Conference Proc. 297 (1993). 